\title{
Hand-assisted laparoscopic surgery for a large gastrointestinal stromal tumor of the stomach
}

\author{
Hiroshi Yano ${ }^{1}$, Yutaka Kimura ${ }^{1}$, Takashi Imazawa ${ }^{1}$, Hirotoshi Takemoto ${ }^{1}$, Mitsunobu Imasato ${ }^{1}$, \\ TAKushi Monden ${ }^{1}$, and Shigeru OKamoto ${ }^{2}$ \\ ${ }^{1}$ Department of Surgery, NTT West Osaka Hospital, 2-6-40 Karasugatsuji, Tennoji-ku, Osaka 543-8922, Japan \\ ${ }^{2}$ Department of Pathology, NTT West Osaka Hospital, Osaka, Japan
}

\begin{abstract}
We report two cases of large gastrointestinal stromal tumor (GIST) of the stomach that were successfully treated by handassisted laparoscopic surgery (HALS). Two patients, a 56year-old woman and a 60-year-old man, were admitted to our department for the treatment of a large submucosal tumor of the stomach. After gastrointestinal endoscopy, ultrasonography, computed tomography, and magnetic resonance imaging, we suspected that the masses, measuring $7.0 \mathrm{~cm}$ and $8.0 \mathrm{~cm}$ in diameter, respectively, were GISTs in the stomach. However, preoperatively, we could not rule out the possibility of malignant neoplasms, because they had been bleeding or gradually growing. Hand-assisted laparoscopic wedge resection was safely performed for the diagnosis and treatment of the submucosal tumor of the stomach. The immunohistochemical diagnosis in both patients was GIST of the stomach with intermediate-grade malignancy. HALS may be a good indication for large GISTs of the stomach that are difficult to diagnose preoperatively, whether they are malignant or benign, because it is safe and minimally invasive, promoting rapid recovery.
\end{abstract}

Key words Gastrointestinal stromal tumor (GIST) · Handassisted laparoscopic surgery (HALS) - Submucosal tumor of the stomach $\cdot$ Wedge resection

\section{Introduction}

Gastrointestinal stromal tumor (GIST) is pathologically defined as a tumor consisting of spindle-shaped cells of mesenchymal origin, developing in the gastrointestinal tract [1,2]. With improved immunohistochemical technology and electron microscopy, it is now clearly shown that GISTs express different histological features, including myogenic properties, neural attributes, or both

Offprint requests to: $\mathrm{H}$. Yano

Received: January 21, 2005 / Accepted: April 5, 2005
$[3,4]$. Recently, the origin of GISTs has been proposed to be the interstitial cell of Cajal, which is the pacemaker cell of the gastrointestinal system [5]. In addition, CD 34 and c-kit protein have been shown to be markers for the interstitial cell of Cajal [5-7]. The majority of GISTs are positive for $\mathrm{CD} 34$, a hematopoietic progenitor cell antigen, and are frequently marked by the presence of the c-kit protooncogene product, a transmembrane tyrosine kinase receptor protein [5-8]. The preoperative diagnosis of GIST is relatively difficult to make, because biopsy samples are rarely obtained during routine gastrofiberscopic procedures. Therefore, resection of the tumor is generally necessary, from both the diagnostic and the therapeutic aspects, in patients with GIST of the stomach. There are variable operative approaches, and most surgeons expect that a laparoscopic procedure will be better than open surgery because it carries a lower risk of complications, faster recovery, less pain, and better cosmesis. The development of endoscopic stapling devices has made laparoscopic wedge resection an interesting and desirable alternative to the conventional open approach. However, a few series of case reports have demonstrated that GIST, as well as other benign tumors, can be resected in this fashion. We report two cases of large GIST of the stomach that were successfully treated by hand-assisted laparoscopic surgery (HALS).

\section{Case reports}

\section{Case 1}

A 56-year-old woman was admitted to our department for investigation of anemia, in December 2003. She had undergone a partial thyroidectomy for thyroid cancer a year earlier and a hysterectomy 6 years earlier, for prolapse of the uterus. Double-contrast study of upper gastrointestinal (GI) series showed a protruding mass 
lesion with deep ulcer formation originating from the greater curvature of the upper portion of the stomach. An upper GI endoscopy showed a submucosal tumor, with ulceration, on the greater curvature of the upper portion of the stomach. A homogeneous low echoic mass, measuring $7.0 \mathrm{~cm}$ in diameter, was found in the fourth layer by endoscopic ultrasonography (US). The pathological diagnosis of the tumor was GIST, suspected from a biopsy specimen obtained by gastroendoscopy. A large homogeneous mass, measuring $7.0 \mathrm{~cm}$ in diameter, was found in the stomach, (with no liver metastasis and no regional lymph node metastasis) by computed tomography (CT) scan and magnetic resonance imaging (MRI; Fig. 1A). The results of laboratory investigations were within normal limits, except for a red blood cell count of $3.19 \times 10^{6}$; hemoglobin, $9.5 \mathrm{~g} / \mathrm{dl}$; and hematocrit, $29.1 \%$. Tumor markers, including the serum concentrations of carcinoembryonic antigen (CEA) and carbohydrate antigen (CA) 19-9, were within normal limits. Hand-assisted laparoscopic wedge resection of the stomach was performed because, preoperatively, we could not rule out the possibility of a malignant neoplasm. The patient's intra- and postoperative courses were uneventful. The duration of surgery was $85 \mathrm{~min}$ and the intraoperative blood loss was $10 \mathrm{~g}$. Histopathologically, the final diagnosis was GIST of the stomach with intermediate-grade malignancy. Oral feeding was started on the second postoperative day. The patient was discharged on postoperative day 13 in excellent condition. She remains well, with no sign of recurrence of GIST, 13 months after her operation.

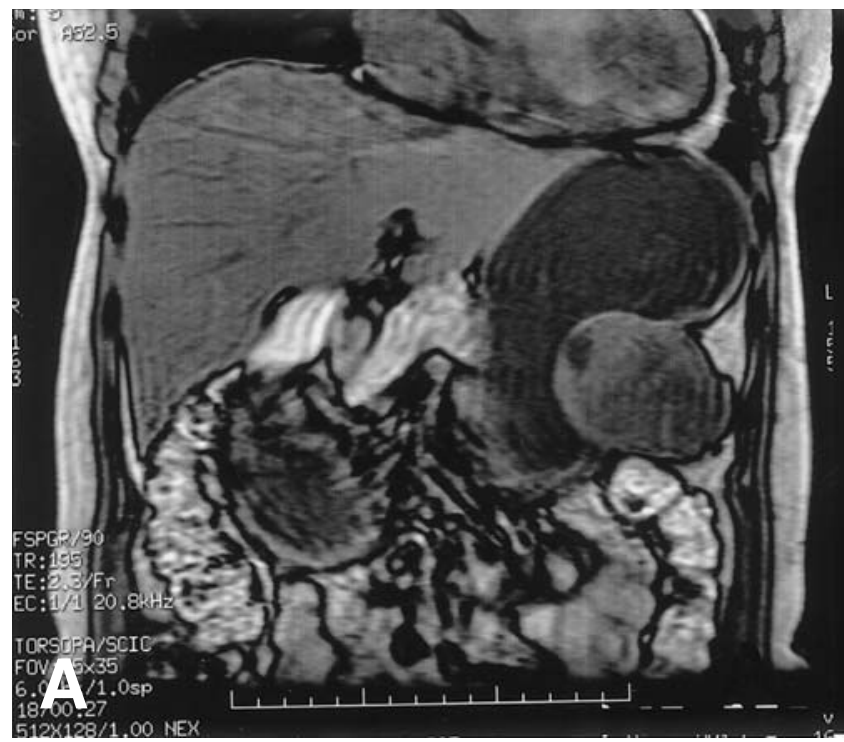

Case 2

A 60-year-old man was admitted to our department for the treatment of a submucosal tumor of the stomach in April 2004. He had been diagnosed as having the submucosal tumor of the stomach by an upper GI endoscopy 3 years previously, and had been followed up. The tumor was gradually growing in size. Gastroendoscopy showed a large submucosal tumor with bridging fold on the anterior wall of the upper-third portion of the stomach. Enhanced CT scan showed a low-density mass, measuring $8.0 \mathrm{~cm}$ in diameter, in the gastric wall, with no liver metastasis and no regional lymph node metastasis (Fig. 1B). Abdominal MRI also revealed the mass, which showed low to isointensity on a T1-weighted image and irregular intensity on a T2weighted image. GIST of the stomach was suspected pathologically from an endoscopic fine-needle aspiration biopsy sample. He had no complaints. Physical examination and results of laboratory investigations, including the serum concentrations of CEA and CA19-9, were unremarkable. Hand-assisted laparoscopic wedge resection of the stomach was performed. His intra- and postoperative courses were uneventful. The duration of surgery was $91 \mathrm{~min}$ and the intraoperative blood loss was $20 \mathrm{~g}$. Histopathologically, the final diagnosis was GIST of the stomach with intermediate-grade malignancy. Oral feeding was commenced on the second postoperative day. The patient was discharged on postoperative day 12 in excellent condition. He remains well, with no sign of recurrence of GIST, 9 months after the surgery.

Fig. 1. A In case 1, abdominal magnetic resonance imaging (MRI) shows a mass, measuring $7.0 \mathrm{~cm}$ in diameter, on the greater curvature of the upper portion of the stomach. B In case 2, an enhanced computed tomography (CT) scan shows a low-density mass, measuring $8.0 \mathrm{~cm}$ in diameter, on the anterior wall of the upper-third portion of the stomach

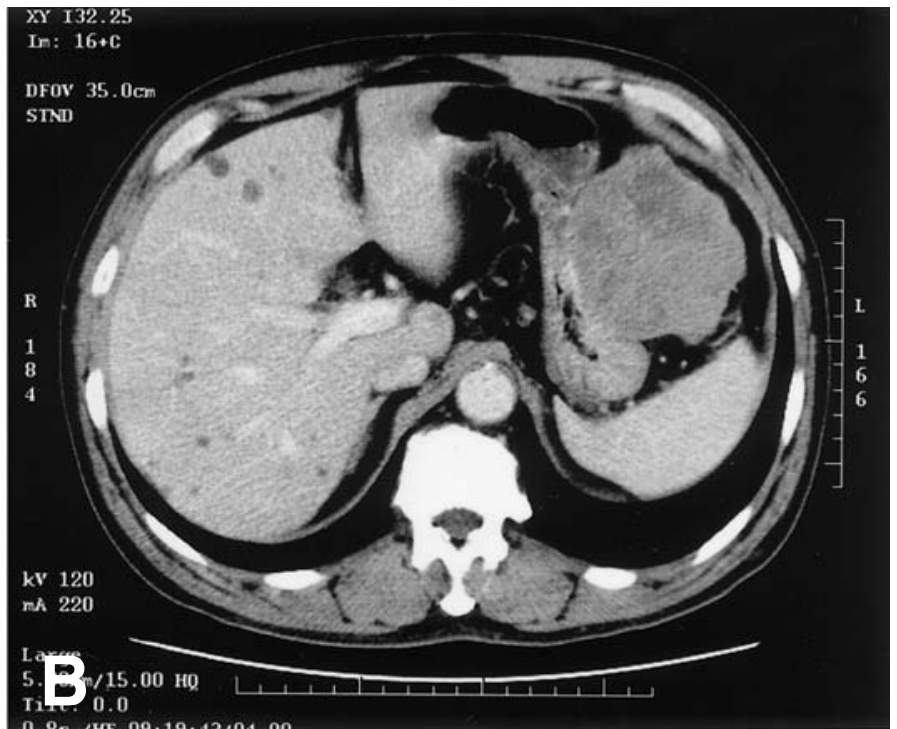




\section{Procedure of HALS for large GIST of the stomach}

Surgery was performed with the patient under general and epidural anesthesia, in the supine position. First, a mini-laparotomy was performed, with a $7-\mathrm{cm}$ skin incision in the mid-upper abdomen. A Lapdisc abdominal wall sealing device (Hakko Medical, Tokyo, Japan) was inserted through the mini-laparotomy wound to prevent the leakage of carbon dioxide gas, and a $10-\mathrm{mm}$ laparoscopic port was inserted through the center of the Lapdisc device. Pneumoperitoneum was maintained with carbon dioxide gas, at a pressure of $10 \mathrm{mmHg}$, during surgery. Three trocars were inserted in the upper abdomen (Fig. 2). Re-placement of the laparoscope through the supraumbilical port for better visualization within the left upper quadrant revealed a huge tumor in the upper portion of the stomach. The stomach, abdominal cavity, and liver were explored with laparoscopic visualization, and we confirmed, macroscopically, that there was no liver metastasis or peritoneal dissemination. The laparoscopic procedure was performed with the surgeon's left hand through the $7-\mathrm{cm}$ skin incision and with electrocautery and laparoscopic coagulating shears (Harmonic Scalpel; Ethicon Endo-Surgery, New Brunswick, NJ, USA) through the port below the costal margin at the left midaxillary line. The tumor was palpated, grasped, and lifted towards the abdominal wall with the fingers of the surgeon's left hand. Then, the tumor was resected with a multifire endoscopic stapling device (Endo GIA II; Tyco Healthcare Japan, Tokyo, Japan), allowing a sufficient distance from the tumor (Fig. 3A). A plastic bag
(Endo Catch II; Tyco Healthcare Japan) was introduced into the abdominal cavity to remove the resected specimen. The plastic bag allowed compression of the soft specimen and extraction through a small incision, without contamination of the wound. The specimen was delivered out of the abdominal cavity through the $7-\mathrm{cm}$ skin incision (Fig. 3B). The skin incisions were sutured in a layer-to-layer fashion.

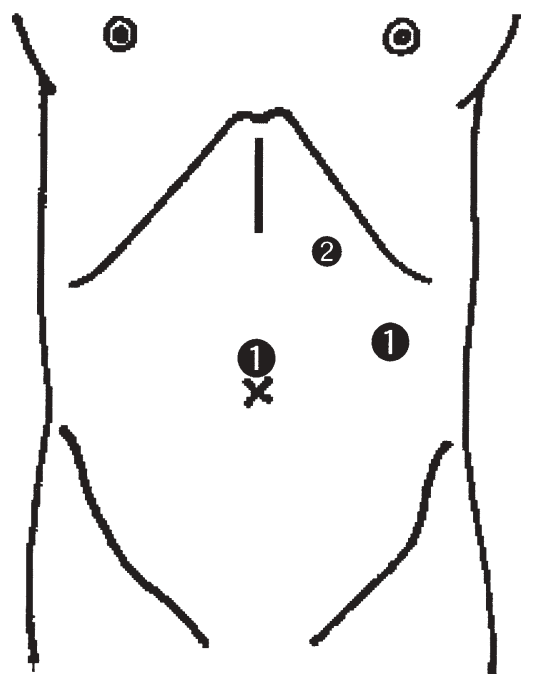

Fig. 2. Trocar placement and mini-laparotomy wound in hand-assisted laparoscopic surgery (HALS) for a large gastrointestinal stromal tumor (GIST) of the stomach. 1, 10-mm trocar; 2, 5-mm trocar
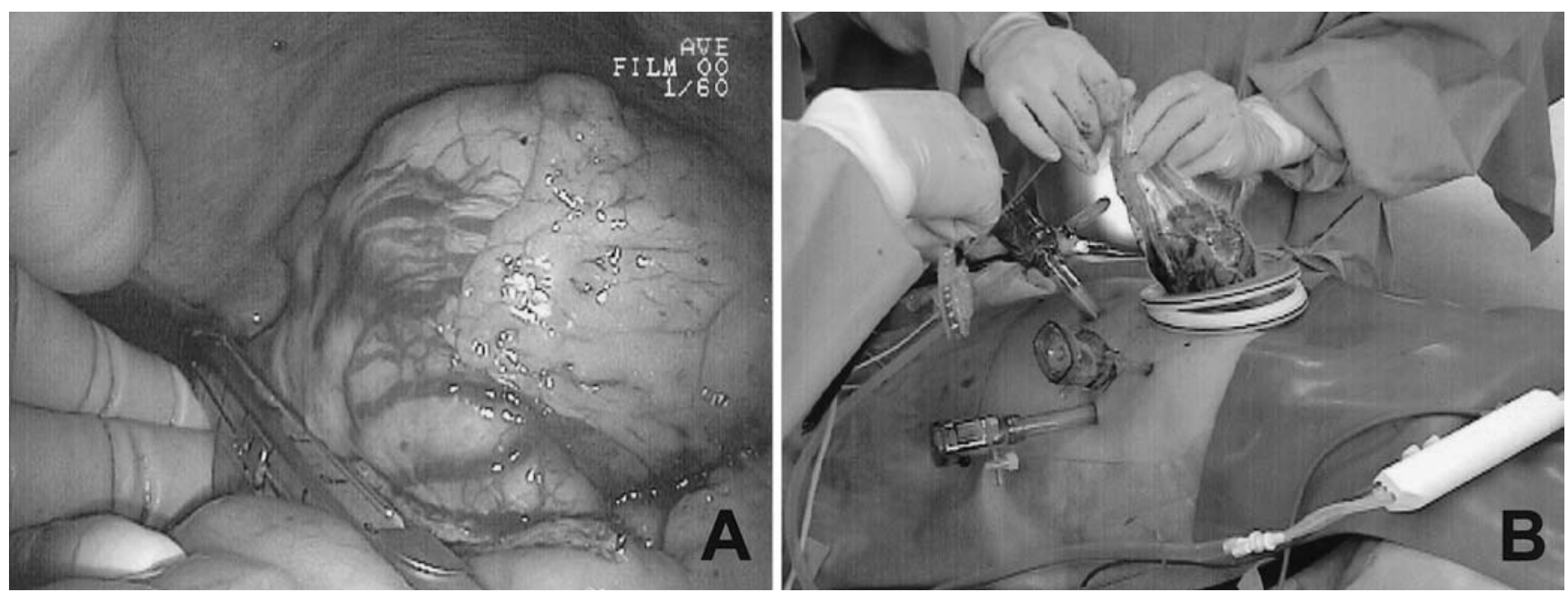

Fig. 3. A Procedure of HALS for large GIST of the stomach. The tumor was palpated, grasped, and lifted towards the abdominal wall with the fingers of the surgeon's left hand. Then, the tumor was resected with a multifire endoscopic stapling device, allowing a sufficient distance from the tumor. B The specimen was delivered out of the abdominal cavity, through a 7-cm skin incision, using a plastic bag 


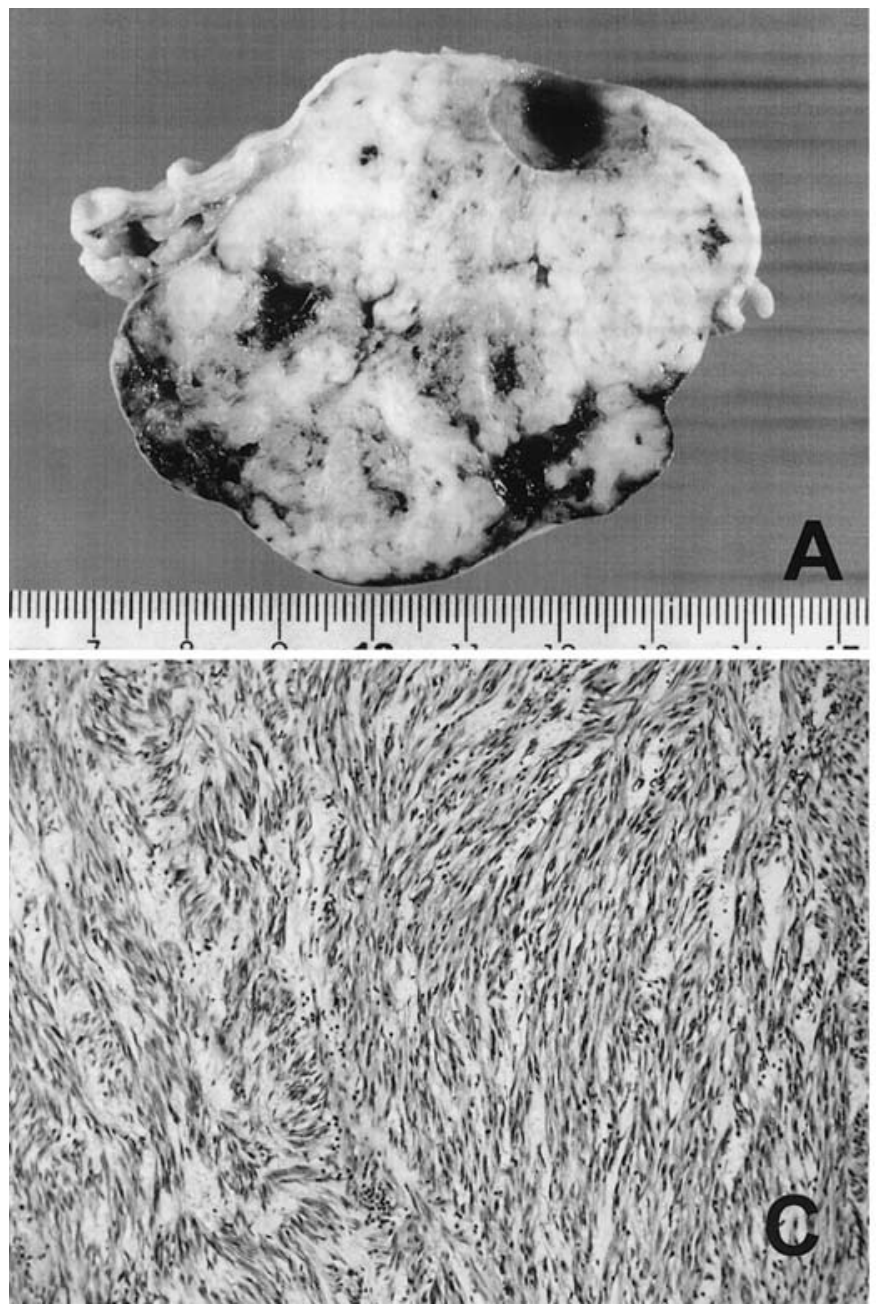

Fig. 4A-D. Pathology. In case 1, the cut surface of the specimen shows a white submucosal tumor with clear margins and ulcer formation (A). In case 2, the tumor mass is attached to the wall of the stomach, and projects into the abdominal cavity (B). Histopathologically, proliferation of spindle cells with

\section{Pathology}

In case 1 , the cut surface of the specimen showed a white submucosal tumor with clear margins (Fig. 4A). In case 2 , the tumor mass was attached to the wall of the stomach, and projected into the abdominal cavity (Fig. 4B). Histopathologically, proliferation of spindle cells with elongated nuclei and eosinophilic cytoplasm was observed in both cases (shown for case 1 in Fig. 4C). The nuclei generally showed round or blunted ends and the chromatin pattern varied from sparse (with inconspicuous nuclei) to hyperchromatic, sometimes pleomorphic prominent nuclei (Fig. 4D). The mitotic activity of the tumor cells was determined by counting the number of apparent mitotic figures in 50 consecutive high-power fields (HPFs; $\times 400)$, in which four to
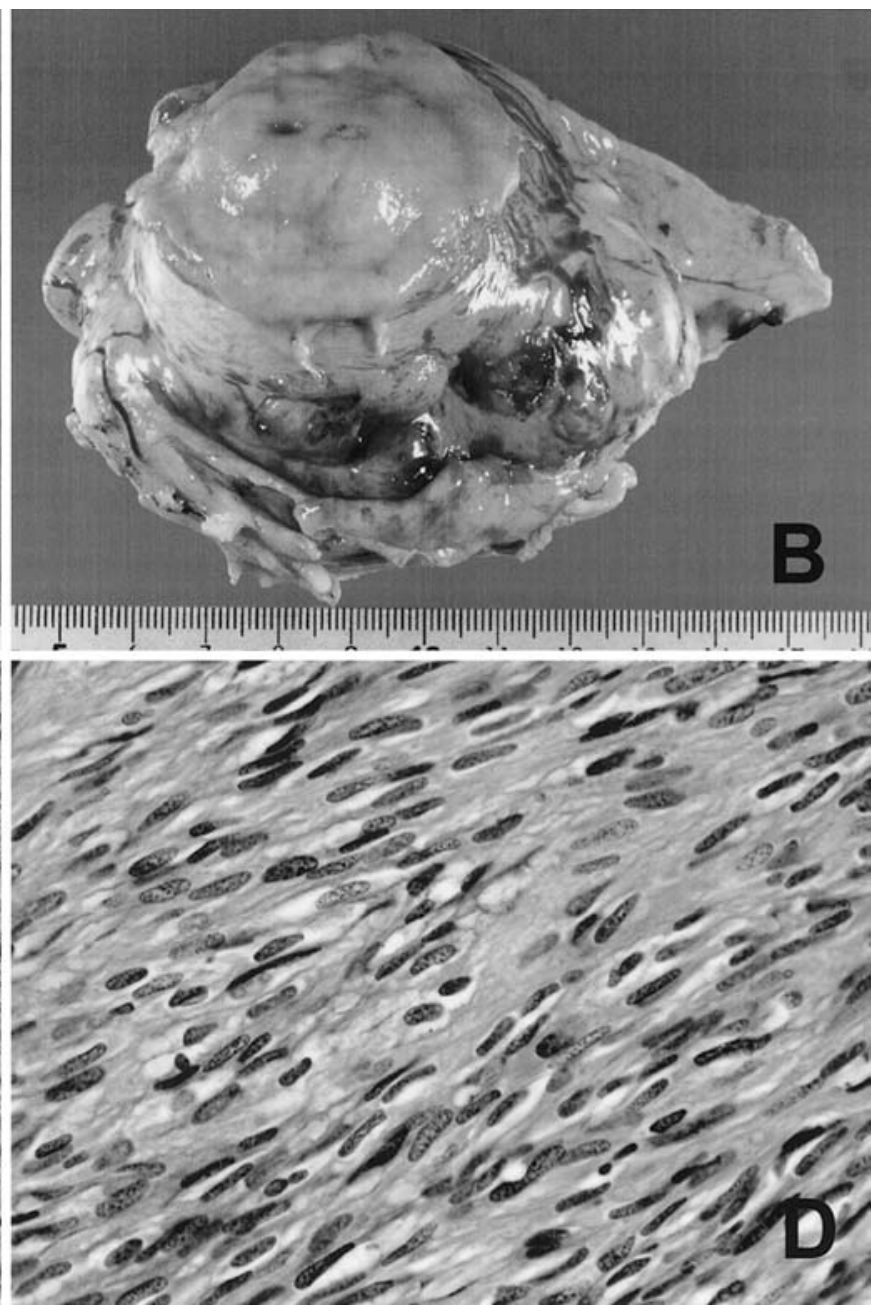

elongated nuclei is observed in case $1(\mathbf{C})$. In case 2, the nuclei show round or blunted ends and the chromatin pattern varies from sparse (with inconspicuous nuclei) to hyperchromatic, sometimes pleomorphic prominent nuclei (D). C H\&E, $\times 100$; D $H \& E, \times 400$

five mitotic figures were found. The results of the immunohistochemical staining in the tumor cells of the two patients are shown in Table 1 . In case 1 , the tumor cells were positive for CD34 and vimentin, and no tumor cell staining was observed for CD117 (c-kit), desmin, smooth-muscle actin (SMA), or S-100. In case 2, the tumor cells were positive for CD34, CD117, vimentin, and S-100. No tumor cell staining was observed for desmin on SMA. All these results suggested that the tumors in both patients should be diagnosed as GIST with intermediate-grade malignancy. 
Table 1. The results of immunohistochemical staining

\begin{tabular}{lcc}
\hline & Case 1 & Case 2 \\
\hline CD34 & + & + \\
CD117 & - & + \\
Vimentin & + & + \\
Desmin & - & - \\
SMA & - & - \\
S-100 & - & + \\
\hline
\end{tabular}

\section{Discussion}

GIST is the most frequent nonepithelial tumor occurring in the stomach and small bowel. In the past, it was considered to be smooth muscle in origin, but recent studies have shown a more complex picture, with evidence of smooth-muscle differentiation, neural differentiation, dual smooth-muscle and neuronal differentiation, or no differentiation. The stomach is the most common organ involved, and size remains the most important predictive factor for its malignant potential $[9,10]$. GISTs may display a wide variety of cell types and histologic growth patterns, including spindle, epithelioid, plasmacytoid, signet ring, granular, and multinucleated cell types [9]. The spindle-cell type is characterized by a proliferation of spindle cells with elongated nuclei and eosinophilic cytoplasm. The histologic features of the tumors in our patients revealed the spindle-cell type of GIST. Immunohistochemically, the hematopoietic progenitor cell antigen, CD34, was found to be expressed in the cells of GISTs in $46 \%$ to $81 \%$ of cases $[5,8]$, and these tumors were frequently marked by the presence of the c-kit protooncogene $[6,7]$. The tumors in our patients showed immunoreactivity for CD34/vimentin and CD34/CD117/vimentin/S-100, respectively. From both the histologic and immunohistochemical findings, the tumors in our patients were diagnosed as GIST of the stomach. Although the majority of GISTs are positive for CD 117 (c-kit), the GIST in case 1 was c-kit-negative. Heinrich et al. [11] report that approximately $35 \%$ of GISTs lacking c-kit mutations have intragenic activation mutations in the related receptor tyrosine kinase, platelet-derived growth factor receptor alpha (PDGFRA). Data for PDGFRA, as well as for c-kit mutation, could have been helpful in making our report more valuable [12]. More than five mitoses per 50 HPFs have been associated in most series with increased metastatic potential and, therefore, with a diagnosis of malignancy $[13,14]$. In our patients, four to five mitotic figures were found in $50 \mathrm{HPFs}$. Size has been, traditionally, one of the most important morphologic parameters associated with prognosis for these tumors. In most studies, the magic number has varied between 5 and $10 \mathrm{~cm}$ in diameter or greater as a cutoff line for separating aggressive from indolent forms $[13,14]$. In our patients, the tumor size was $7.0 \mathrm{~cm}$ and $8.0 \mathrm{~cm}$, respectively, in diameter. According to the Risk Assessment Classification Scheme to Assess Prognosis of Primary Gastrointestinal Stromal Tumors, the tumors in our patients were classified as the intermediate risk group (7-8cm, 4 to 5/50 HPFs) [15]. These data may be regarded as an indication of aggressive potential and warrant the designation of GIST. Size, however, is not an absolute parameter, as rare tumors smaller than $5 \mathrm{~cm}$ have occasionally been observed to behave aggressively and metastasize.

GIST of the stomach is difficult to diagnose preoperatively, no matter whether it is malignant or benign. Although recent advances in imaging techniques, such as US, CT, and MRI, have aided in the identification of space-occupying lesions of the stomach, these techniques do not permit preoperative diagnosis of these lesions. Therefore, resection of the tumor is generally necessary, from both the diagnostic and the therapeutic aspects, in patients with GIST of the stomach. The recurrent or metastatic form of the usual type of gastric cancer is lymph node metastasis, whereas most malignant submucosal tumors exhibit distant metastasis $[16,17]$. This finding suggests that lymphadenectomy is not necessary for malignant gastric submucosal tumors, including GISTs. Considering these tumor characteristics, local resection might be the first choice of treatment. Laparoscopic wedge resection of the stomach is warranted as a curative and minimally invasive surgery for both diagnosis and treatment of GISTs. The development of endoscopic stapling devices has made laparoscopic wedge resection an interesting and desirable alternative to the conventional open approach. Laparoscopic wedge resection for a small-size GIST has been performed in a few series of case reports $[18,19]$. This method seems to be a safe, oncologically adequate technique to remove GISTs of the stomach, and should be investigated further because of its potential advantages, in terms of reduced postoperative pain, earlier oral intake, shorter hospital stay, and improved cosmesis. However, there are no previous reports of laparoscopic treatment for a large GIST of the stomach. HALS has developed as an advanced laparoscopic technique, because use of the hand allows for easy exposure, complete exploration, meticulous dissection, and immediate hemostasis [20]. Wedge resection, with a free margin of $2-3 \mathrm{~cm}$, is considered to be a suitable treatment for benign tumors and, in particular, for GIST of the stomach [21], and enucleation of the stomach is an inappropriate method for GIST. Tactile sensing and adequate traction of the tumor are necessary for the resection of a large GIST of the stomach to detect the limits of the tumor, which are usually obscured from the laparoscopic view. In view of these facts, HALS 
is a more suitable procedure (with sufficient surgical margins) than pure laparoscopic surgery to resect a large GIST of the stomach. One of the advantages of HALS compared to pure laparoscopic surgery is the gentle manipulation of the tumor by hand, which can decrease the risk of tumor dissemination during surgery. Moreover, HALS can retain many of the recovery advantages of pure laparoscopic procedures.

Recurrence of GIST is usual, and one report found only $10 \%$ of patients to be free of disease after longterm follow-up [16]. Recurrence tends to involve the peritoneal surface or the liver. Although approximately half of the patients with primary disease who undergo complete resection will survive for 5 years, surgery alone is inadequate for GISTs. The high rate of local and distant recurrence underscores the need for adjuvant therapy, but an effective adjuvant therapy has not been available. Imatinib (the tyrosine kinase inhibitor STI571) has recently been found to have a dramatic antitumor effect on GISTs. It has proven to be effective in metastatic GIST and is also under investigation as neoadjuvant and adjuvant therapy [22-24].

The most serious objection that has arisen regarding laparoscopic surgery is the concern following reports of port-site recurrence in patients with malignant tumors [25-28]. Such recurrences occur when trocars are pulled out, allowing a contaminated instrument, laden with tumor cells, to pass unprotected through the abdominal wall, but the pathogenesis and natural history of these recurrences are still unclear. In our patients, with no adjuvant therapy, although no signs of local recurrence, metastasis to other organs, or port-site recurrence have been found after surgery, further follow-up will be necessary.

In conclusion, HALS may be a good indication for large GISTs of the stomach that are difficult to diagnose preoperatively, whether they are malignant or benign, because it is safe and minimally invasive, promoting rapid recovery.

Acknowledgments The authors thank technicians Mr. Masahide Sakai and Mr. Shogo Shimoyama for their assistance with the immunohistochemical staining of the tumor cells.

\section{References}

1. Miettinen M. Gastrointestinal stromal tumors - an immunohistochemical study of cellular differentiation. Am J Clin Pathol 1988;89:601-10.

2. Pike AM, Lloyd RV, Appelman HD. Cell markers in gastrointestinal stromal tumors. Hum Pathol 1988;19:830-4.

3. Herrera GA, Cerezo L, Jones JE, Sack J, Grizzle WE, Pollack WJ, et al. Gastrointestinal autonomic nerve tumors: "plexosarcoma". Am J Surg Pathol 1989;17:846-53.
4. Lauwers GY, Erlandson RA, Casper ES, Brennan MF, Woodruff JM. Gastrointestinal autonomic nerve tumors. A clinicopathological, immunohistochemical, and ultrastructual study of 12 cases. Am J Surg Pathol 1993;17:887-97.

5. Kindblom LG, Remotti HE, Aldenborg F, Meis-Kindblom JM. Gastrointestinal pacemaker cell tumor (GIPACT): gastrointestinal stromal tumors show phenotypic characteristics of the interstitial cells of Cajal. Am J Pathol 1998;152:1259-69.

6. Hirota S, Isozaki K, Moriyama $\mathrm{Y}$, Hashimoto $\mathrm{K}$, Nishida $\mathrm{T}$, Ishiguro S, et al. Gain-of-function mutations of c-kit in human gastrointestinal stromal tumors. Science 1998;279:577-80.

7. Sarlomo-Rikala M, Kovatich A, Barusevicius A, Miettinen M. CD 117: a sensitive marker for gastrointestinal stromal tumors that is more specific than CD 34. Mod Pathol 1998;11:728-34.

8. Miettinen M, Virolainen M, Maarit SR. Gastrointestinal stromal tumors - value of CD 34 antigen in their identification and separation from true leiomyomas and schwannomas. Am J Surg Pathol 1995;19:207-16.

9. Suster S. Gastrointestinal stromal tumors. Semin Diagn Pathol 1996;13:297-313.

10. DeMatteo RP, Lewis JJ, Leung D, Mudan SS, Woodruff JM, Brennan MF. Two hundred gastrointestinal stromal tumors. Ann Surg 2000;231:51-8.

11. Heinrich MC, Corless CL, Duensing A, McGreevey L, Chen CJ, Joseph N, et al. PDGFRA activating mutations in gastrointestinal stromal tumors. Science 2003;299:708-10.

12. Medeiros F, Corless CL, Duensing A, Hornick JL, Oliveira AM, Heinrich MC, et al. KIT-negative gastrointestinal stromal tumors: proof of concept and therapeutic implications. Am J Surg Pathol 2004;28:889-94.

13. Grant CS, Kim CH, Farrugia G, Zinsmeister A, Goellner JR. Gastric leiomyosarcoma. Prognostic factors and surgical management. Arch Surg 1991;126:985-90.

14. Amin MB, Ma CK, Linden MD, Kubus JJ, Zarbo RJ. Prognostic value of proliferating cell nuclear antigen index in gastric stromal tumors. Correlation with mitotic count and clinical outcome. Am J Clin Pathol 1993;100:428-32.

15. Demetri GD, Benjamin R, Blanke CD, Choi H, Corless C, DeMatteo RP, et al. NCCN task force report: optimal management of patients with gastrointestinal stromal tumor (GIST) expansion and update of NCCN clinical practice guidelines. JNCCN 2004;2 (Suppl 1):1-26.

16. Ng EH, Pollock RE, Romsdahl MM. Prognostic implications of patterns of failure for gastrointestinal leiomyosarcomas. Cancer 1992;69:1334-41.

17. Salmela H. Smooth muscle tumors of the stomach. A clinical study of 112 cases. Acta Chir Scand 1968;134:384-91.

18. Kimata M, Kubota T, Otani Y, Ohgami M, Ishikawa Y, Yokoyama T, et al. Gastrointestinal stromal tumors treated by laparoscopic surgery: report of three cases. Surg Today 2000; 30:177-80.

19. Shirai H, Takeuchi T, Naka T, Minaghi S, Kimura A, Hamazaki S, et al. Gastrointestinal stromal tumor of the stomach: report of a case. Surg Today 2001;31:346-9.

20. Romanelli JR, Kelly JJ, Litwin DEM. Hand-assisted laparoscopic surgery in the United States: an overview. Semin Laparosc Surg 2001;8:96-103.

21. Llorente J. Laparoscopic gastric resection for gastric leiomyoma. Surg Endosc 1994;8:887-9.

22. Joensuu H, Roberts PJ, Sarlomo-Rikala M, Andersson LC, Tervahartia P, Tuveson D, et al. Effect of the tyrosine kinase inhibitor STI571 in a patient with a metastatic gastrointestinal stromal tumor. N Engl J Med 2001;344:1052-6.

23. Yamamoto S, Kubo S, Shuto T, Yamamoto T, Hirohashi K, Tanaka H, et al. Treatment with STI571, a tyrosine kinase inhibitor, for gastrointestinal stromal tumor with peritoneal dissemination and multiple liver metastasis. J Gastroenterol 2003;38:896-9.

24. Kanda T, Ohashi M, Makino S, Kaneko K, Matsuki A, Nakagawa $\mathrm{S}$, et al. A successful case of oral molecularly targeted therapy 
with imatinib for peritoneal metastasis of a gastrointestinal stromal tumor. Int J Clin Oncol 2003;8:180-3.

25. Cirocco WC, Schwartzman A, Golub RW. Abdominal wall recurrence after laparoscopic colectomy for colon cancer. Surgery 1994;116:842-6.

26. Fusco MA, Paluzzi MW. Abdominal wall recurrence after laparoscopic-assisted colectomy for adenocarcinoma of the colon. Report of a case. Dis Colon Rectum 1993;36:858-61.
27. Ortega AE, Beart RW Jr, Steele GD Jr, Winchester DP, Greene FL. Laparoscopic bowel surgery registry. Preliminary results. Dis Colon Rectum 1995;38:681-6.

28. Shoup M, Brennan MF, Karpeh MS, Gillern SM, McMahon RL, Conlon K. Port site metastasis after diagnostic laparoscopy for upper gastrointestinal tract malignancies: an uncommon entity. Ann Surg Oncol 2002;9:632-6. 